\title{
Elevated markers of thrombo-inflammatory activation predict outcome in patients with cardiovascular comorbidities and COVID-19 disease: insights from the LEOSS registry
}

\author{
Sebastian Cremer ${ }^{1,2,3} \cdot$ Carolin Jakob $^{4} \cdot$ Alexander Berkowitsch $^{1} \cdot$ Stefan Borgmann $^{5} \cdot$ Lisa Pilgram $^{12}$. \\ Lukas Tometten $^{6} \cdot$ Annika Classen $^{4} \cdot$ Kai Wille $^{7} \cdot$ Simon Weidlich $^{8} \cdot$ Beate Gruener $^{9} \cdot$ Stefanie Dimmeler $^{2,3,10}$. \\ Steffen Massberg ${ }^{11}$. Siegbert Rieg ${ }^{13}$. Andreas M. Zeiher ${ }^{1,2,3}$ on behalf of the LEOSS study group
}

Received: 12 August 2020 / Accepted: 21 October 2020 / Published online: 19 November 2020

(c) The Author(s) 2020

\begin{abstract}
Aims SARS-CoV-2 infection is associated with adverse outcomes in patients with cardiovascular disease. Here, we analyzed whether specific biomarkers predict the clinical course of COVID-19 in patients with cardiovascular comorbidities.

Methods and results We enrolled 2147 patients with SARS-CoV-2 infection which were included in the Lean European Open Survey on SARS-CoV-2 (LEOSS)-registry from March to June 2020. Clinical data and laboratory values were collected and compared between patients with and without cardiovascular comorbidities in different clinical stages of the disease. Predictors for mortality were calculated using multivariate regression analysis. We show that patients with cardiovascular comorbidities display significantly higher markers of myocardial injury and thrombo-inflammatory activation already in the uncomplicated phase of COVID-19. In multivariate analysis, elevated levels of troponin [OR 1.54; (95\% CI 1.22-1.96), $p<0.001$ )], IL-6 [OR 1.69 (95\% CI 1.26-2.27), $p<0.013)$ ], and CRP [OR 1.32; (95\% CI 1.1-1.58), $p<0.003$ )] were predictors of mortality in patients with COVID-19.

Conclusion Patients with cardiovascular comorbidities show elevated markers of thrombo-inflammatory activation and myocardial injury, which predict mortality, already in the uncomplicated phase of COVID-19. Starting targeted anti-inflammatory
\end{abstract}

Siegbert Rieg and Andreas M. Zeiher have shared last authorship.

Electronic supplementary material The online version of this article (https://doi.org/10.1007/s00392-020-01769-9) contains supplementary material, which is available to authorized users.

Andreas M. Zeiher

zeiher@em.uni-frankfurt.de

1 Department of Medicine III, Cardiology/Angiology/

Nephrology, Goethe University of Frankfurt, Theodor-Stern-Kai 7, 60590 Frankfurt, Germany

2 German Center for Cardiovascular Research DZHK, partner site Rhine-Main, Berlin, Germany

3 Cardiopulmonary Institute, Goethe University Frankfurt, Frankfurt, Germany

4 Faculty of Medicine and University Hospital Cologne, Department I for Internal Medicine, University of Cologne, Cologne, Germany

5 Department of Infectious Diseases and Infection Control, Ingolstadt Hospital, Ingolstadt, Germany

6 Hospital Ernst Von Bergmann, Potsdam, Germany
7 Department of Hematology/Oncology, Johannes Wesling Hospital, Minden, Germany

8 Department of Internal Medicine II, University Hospital Rechts Der Isar, Technical University Munich, Munich, Germany

9 Department of Infectious Diseases, Internal Medicine III, Ulm, Germany

10 Institute for Cardiovascular Regeneration, Goethe University Frankfurt, Frankfurt, Germany

11 Department of Medicine I, Klinikum Der Universität München, Munich, Germany

12 Department of Internal Medicine, Hematology/Oncology, Goethe University Frankfurt, Frankfurt am Main, Germany

13 Internal Medicine II, Department of Infectious Diseases, Freiburg University Hospital, Freiburg, Germany 
therapy and aggressive anticoagulation already in the uncomplicated phase of the disease might improve outcomes after SARS-CoV-2 infection in patients with cardiovascular comorbidities.

\section{Graphic abstract}

Elevated markers of thrombo-inflammatory activation predict outcome in patients withcardiovascular comorbidities and COVID-19 disease: insights from the LEOSS registry

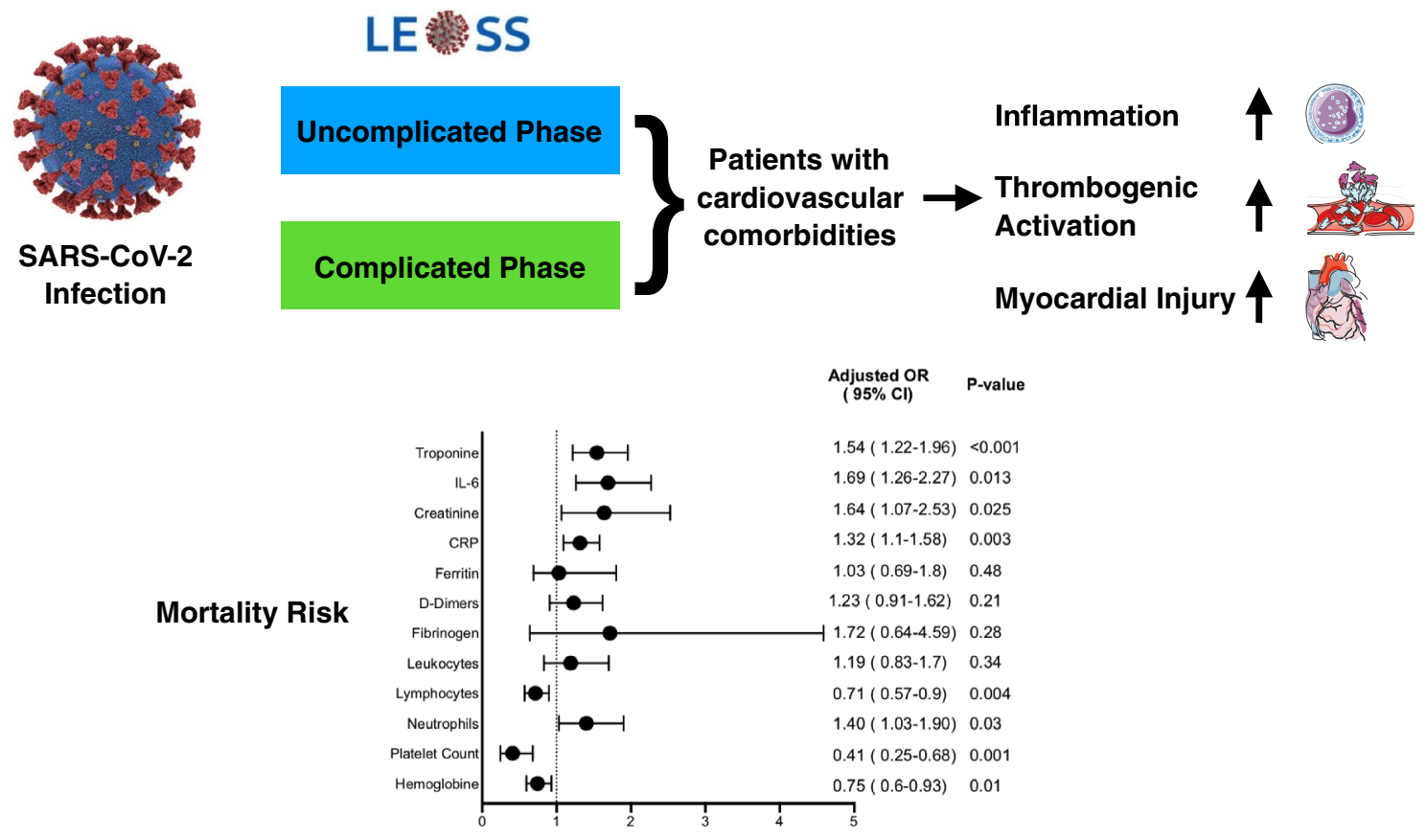

Keywords Inflammation $\cdot$ COVID-19 $\cdot$ Myocardial injury $\cdot$ Coagulation $\cdot$ Mortality

\section{Introduction}

Infection with the novel coronavirus SARS-CoV-2 causes the so-called coronavirus disease COVID-19, which has become a worldwide pandemic [1]. Based on initial reports from the outbreak of the disease in China, COVID-19 progression is characterized by three distinct phases: an initial infection phase followed by a respiratory distress phase and finally culminating in a severe hyperinflammatory state [2]. While more than $80 \%$ of SARS-CoV-2 infections show only mild or even absent symptoms, the cardiovascular system has been documented to play an important role both as a primary target as well as a risk amplifying comorbidity factor for COVID-19 [3-6]. In fact, a very recent study with more than 2700 laboratory confirmed COVID-19 patients revealed that patients with preexisting cardiovascular disease (CVD) are more likely to experience myocardial injury as measured by increased troponin release, which by itself is associated with a profound increase in risk for mortality from COVID-19 [7]. However, major gaps remain to understand the pathophysiological mechanisms, by which infection with
SARS-CoV-2 leads to cardiovascular morbidity and associates with worse clinical outcome in patients with preexisting cardiovascular disease.

Therefore, it was the aim of the present study to decipher specific properties of patients with cardiovascular disease suffering from COVID-19 and to address whether these characteristics contribute to worse clinical outcome during COVID-19 progression. Specifically, we were interested in finding out if cardiac and inflammatory biomarkers in patients with COVID-19 would predict disease outcomes. To do so, we used data from one of the largest European COVID-19 registries, the Lean European Open Survey on SARS-CoV-2 (LEOSS) registry (www.leoss.net) [8].

\section{Methods}

\section{Study population}

The study population consists of 2147 consecutive patients, who were included in the LEOSS (Lean European Open 
Survey on SARS-CoV-2) registry between $03 / 18 / 2020$ and 06/08/2020 across 122 hospitals in Europe, most of them in Germany. A map with all recruiting centers is shown in Fig. 1. All patients had a diagnosis confirmed by positive results of PCR testing. Data collection was performed retrospectively and anonymously, while only data from standard of care treatment are documented. Patients were stratified into the following groups at an initial positive test result for SARS-CoV-2: patients in the uncomplicated phase were either asymptomatic, and had symptoms of upper respiratory tract infection, fever or nausea, emesis, or diarrhea. Patients in the complicated phase had at least one of the following characteristics: new need for oxygen supplementation or clinically relevant increase of prior oxygen home therapy, $\mathrm{PaO} 2$ at room air $<70 \mathrm{mmHg}, \mathrm{SO} 2$ at room air $<90 \%$, increase of AST or ALT $>5 \times$ ULN (upper limit of normal), new cardiac arrythmia, new pericardial effusion $>1 \mathrm{~cm}$ or new heart failure with pulmonary edema, congestive hepatopathy, or peripheral edema. Patients in the critical phase were dependent on catecholamines, experienced life-threatening cardiac arrhythmia, had mechanical ventilation (invasive or non-invasive), or need for unplanned mechanical ventilation prolongation ( $>24 \mathrm{~h}$ ) of planned mechanical ventilation, liver failure with an INR $>3.5$ (quick $<50 \%$ ), a qSOFA score of $>=2$, or acute renal failure with need of dialysis. This study was approved by the responsible ethics committee of all participating study sites.

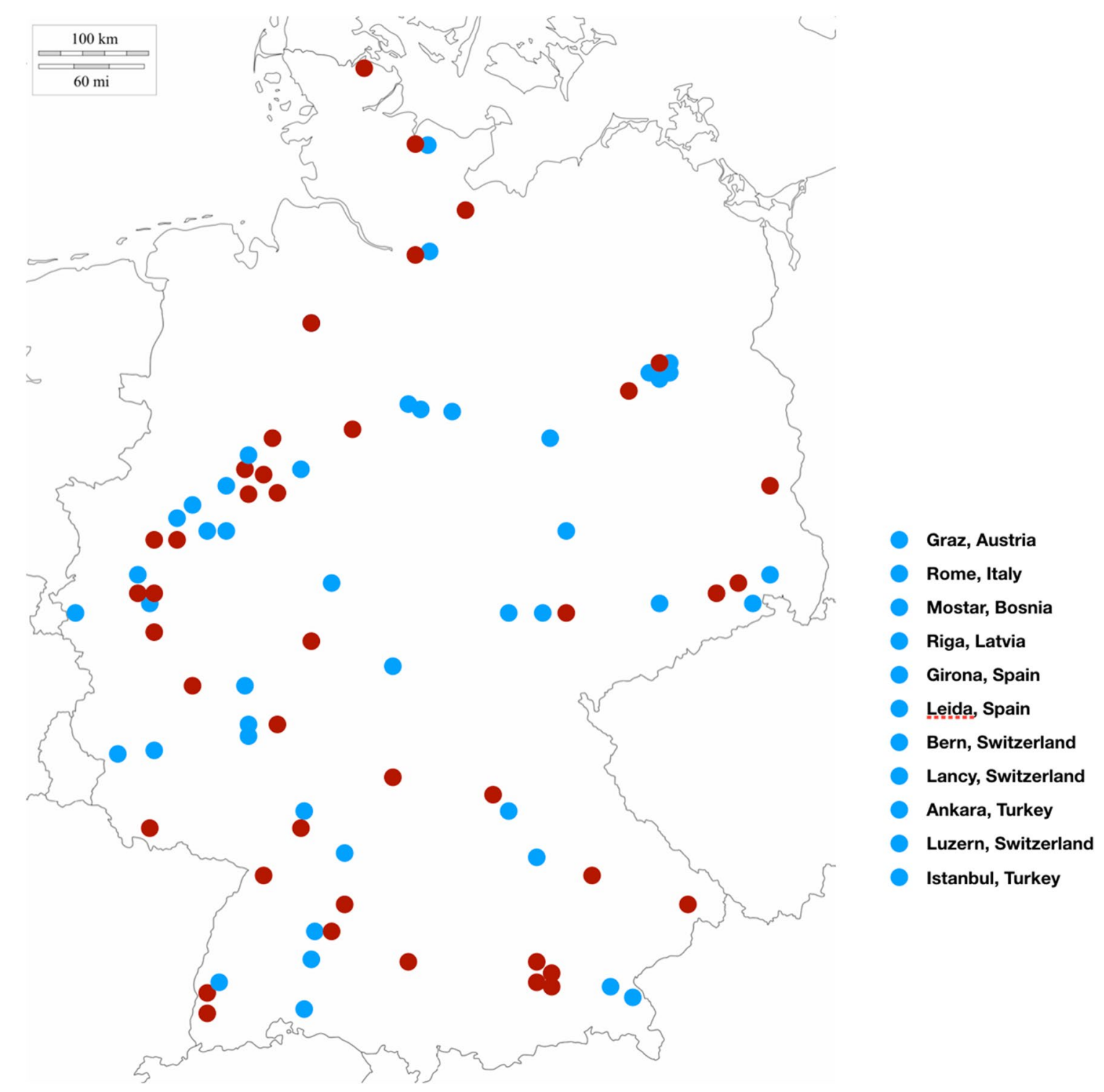

Fig. 1 Map with all centers which added patients to the study cohort. Centers which included patients with cardiovascular comorbidities are highlighted in red 


\section{Data collection}

Demographic, clinical, laboratory, treatment, and outcome data were extracted from the in-hospital medical records. Analyzed laboratory data were collected within $48 \mathrm{~h}$ of a positive PCR result irrespective of the patient's status. Therefore, all biomarker measurements represent baseline values. Data were distributed into different categories. Cardiovascular (CV) comorbidity was defined by one of the following diseases: coronary artery disease, prior myocardial infarction, chronic heart failure, atrial fibrillation, AV-Block, and aortic valve stenosis. As all data are based on anonymous reports, patients can be included without informed consent. Several steps are taken to prevent re-identification which include vertical (categorical assessment of numerical variables) and horizontal data aggregation (data aggregation within the phases of disease). The median of documented cases per center is seven in the entire cohort (range 1-181) and eight (range 3-60) in the subset of patients with cardiovascular comorbidities.

\section{Statistical analysis}

All data are presented as categorical variables (values and proportions) and were analyzed with Chi-square or Fisher's exact test, where appropriate. Outcome in patients being in the non-critical phase at baseline was analyzed with univariate binary regression analysis and parameters associated with outcome were further included in multivariate analysis for identification of independent predictors. The multivariate analysis was performed using logistic regression according to Wald approach in step-down mode. Primary endpoint of the study was death of any cause. Death or reaching a critical condition in patients being not critical when testing positive for SARS-CoV-2 was considered as combined endpoint. For all the statistical analyses, $p<0.05$ was considered to be significant. Analyses were performed with SPSS, version 26 (IBM, Chicago, Illinois).

\section{Results}

\section{Characteristics of patients at baseline}

First, patients were stratified according to disease stage after being tested positive for SARS-CoV-2. Among a total of 2147 patients, $1,343(62.55 \%)$ were in the uncomplicated phase of COVID-19, 641 (29.86\%) in the complicated phase and $163(7.59 \%)$ patients in the critical phase. Increased disease severity correlated with age $(p<0.001)$, which was associated with male gender $(p=0.021)$ and a higher BMI $(p<0.001)$. Of note, patients who presented in a complicated or critical phase of the disease were more likely to suffer from cardiovascular comorbidities $(p<0.001)$, including coronary artery disease $(p=0.011)$, chronic heart failure $(p=0.001)$, and atrial fibrillation $(p=0.001)$. In addition, they were more likely to have hypertension, diabetes mellitus, chronic kidney disease ( $p<0.001$ for all), or a history of cancer $(p=0.043)$, and were more often smokers $(p=0.032$; Table 1).

Next, we compared inflammatory and cardiovascular biomarkers within patients who presented in the uncomplicated, the complicated, and the critical phase, respectively. Here, we report higher levels of IL-6, CRP, ferritin, leukocytes, and neutrophils in patients with progressed COVID-19 disease $(p<0.001$ for all). Lymphocyte levels $(p<0.001)$ and hemoglobin values $(p=0.045)$ were lower in more severe cases. Interestingly, also markers of cardiac damage and thrombogenic activation were elevated in cases with severe COVID-19 infection. Specifically, we report higher levels for troponin, fibrinogen, and D-dimers ( $p<0.001$ for all, Fig. 2).

\section{Biomarkers in patients with cardiovascular disease and COVID-19 according to disease phase}

Hyperinflammation is a hallmark of severe COVID-19 disease, in which myocardial injury occurs frequently and predicts death. Patients with preexisting cardiovascular disease have worse outcomes in COVID-19 [7]. Therefore, we compared biomarkers in the different phases of the disease between patients with cardiovascular comorbidities and patients without an underlying cardiovascular condition. Here, we did not detect significant differences in biomarker levels in the critical phase, except higher CRP values in patients with CVD (Fig. 3). However, in the complicated phase, markers of myocardial injury (troponin, $p<0.001$ ), kidney damage (creatinine, $p<0.001$ ), and thrombogenic activation (D-dimers, $p=0.001$ ) were elevated in patients with CVD, whereas markers of inflammation did not differ (Fig. 4). In the uncomplicated phase, when patients were either asymptomatic or had mild respiratory symptoms, this was very different. Here, patients with cardiovascular comorbidities were more likely to have elevated levels of IL-6 ( $p=0.033)$, CRP $(p=0.026)$, and higher leukocyte counts $(\mathrm{p}=0.014)$ in addition to markers of myocardial injury (Fig. 5). This indicates that patients with underlying cardiovascular disease are more likely to display a systemic inflammatory state already in the uncomplicated phase of the disease. In addition, D-dimers were significantly increased in these patients indicating activation of intravascular coagulation (Fig. 5).

\section{Outcomes and predictors}

Of the 2147 patients included in this analysis, 355 (16.53\%) died during follow-up. $9.08 \%$ (122) of patients who 


\section{Biomarkers in COVID-19 stratified to phases}

Uncomplicated Phase, $n=1343$

Complicated Phase, $n=641$

Critical Phase, $n=163$

A $\quad \begin{gathered}\text { Troponin } \\ p<0.001 \\ \mathrm{n}=483 / 271 / 91\end{gathered}$

Troponin
$p<0.001$
0
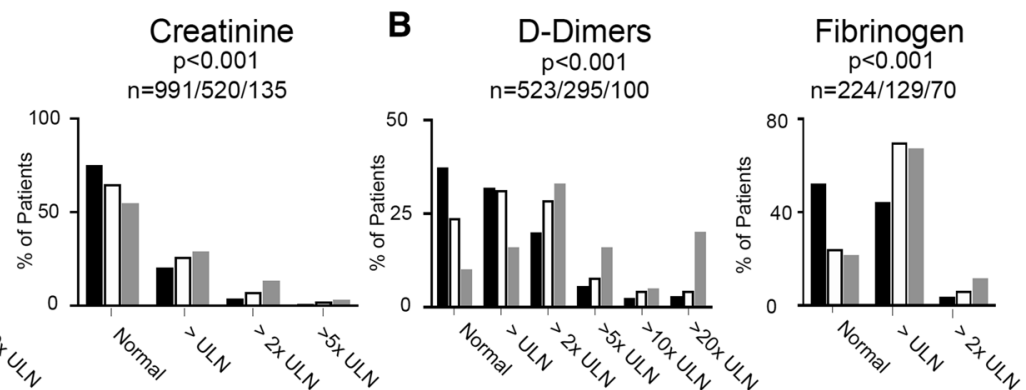

C
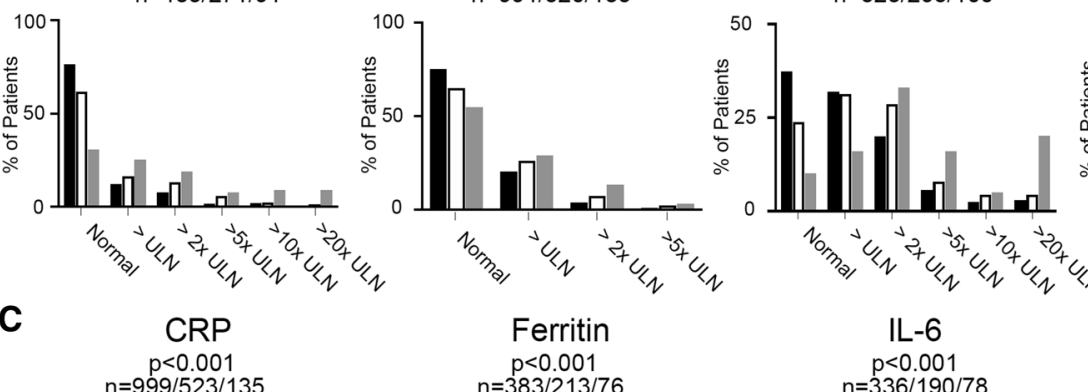

CRP
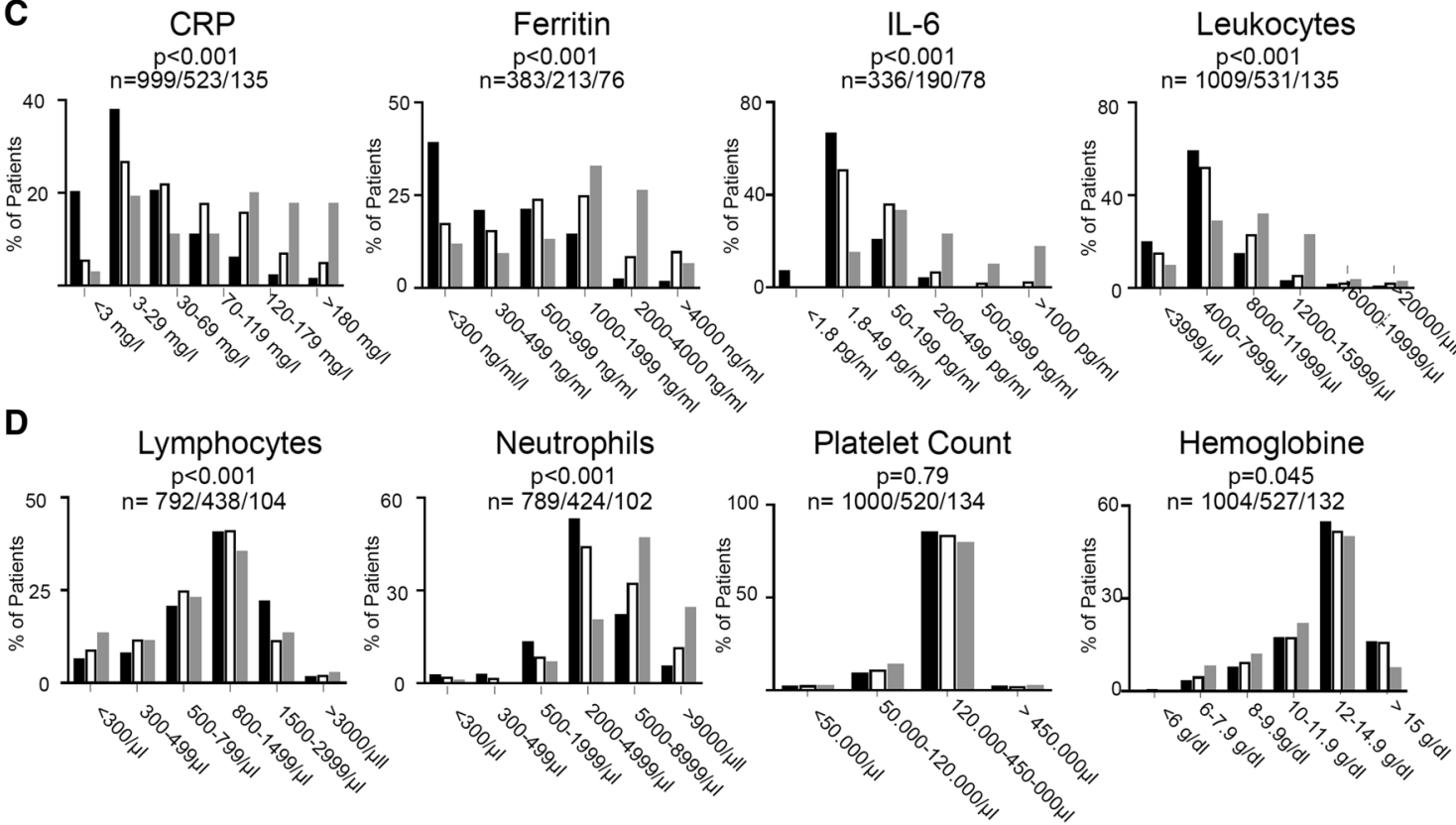

Fig. 2 Serum levels of Troponin and Creatinine (a), D-dimers and Fibrinogen (b), CRP, IL-6, Ferritin, and WBC (c), Lymphocyte and Neutrophil counts, platelet count, and hemoglobin (d) of patients in the whole-study cohort who presented in the uncomplicated phase

(black bars), the complicated phase (white bars), and the critical phase (gray bars). n-numbers of available lab values in each group and $p$ values are depicted in each panel

presented in the uncomplicated phase of the disease died, whereas $24.8 \%$ (159) and $45.4 \%$ (74) of patients who were initially in the complicated and critical phase at baseline, respectively, died.

To analyze the risk factors for mortality in patients, who presented in a non-critical phase of COVID-19, univariate binary regression analysis was performed. Here, higher disease severity, sex and age, cardiovascular comorbidity, hypertension, carotid and peripheral artery disease, as well as diabetes mellitus, chronic kidney disease, pulmonary disease, and a history of cancer were associated with an increased risk of death. In this analysis, all biomarkers

evaluated qualified to assess mortality risk. To define independent predictors of death in patients with COVID-19, multivariate analysis including clinical severity stage was conducted. Here, only a complicated phase of COVID-19 at baseline [OR 1.93 (95\% CI 1.10-3.39); $p<0.001$ ] and the demographic variables sex [OR 0.42 (95\% CI $0.27-0.77$ ); $p=0.006$ ] and age [OR $1.71(95 \%$ CI $1.37-2.14) ; p<0.001]$ were independent predictors of mortality, whereas all comorbidities, including cardiovascular disease, lost their predictive power (Fig. 6a).

Among biomarkers, elevated troponin levels were the strongest predictor of death [OR 1.54; (95\% CI 1.22-1.96); 


\section{Biomarkers in COVID-19 Critical Phase}

- No CV Comorbidity, $\mathrm{n}=110$

CV Comorbidity, $n=53$
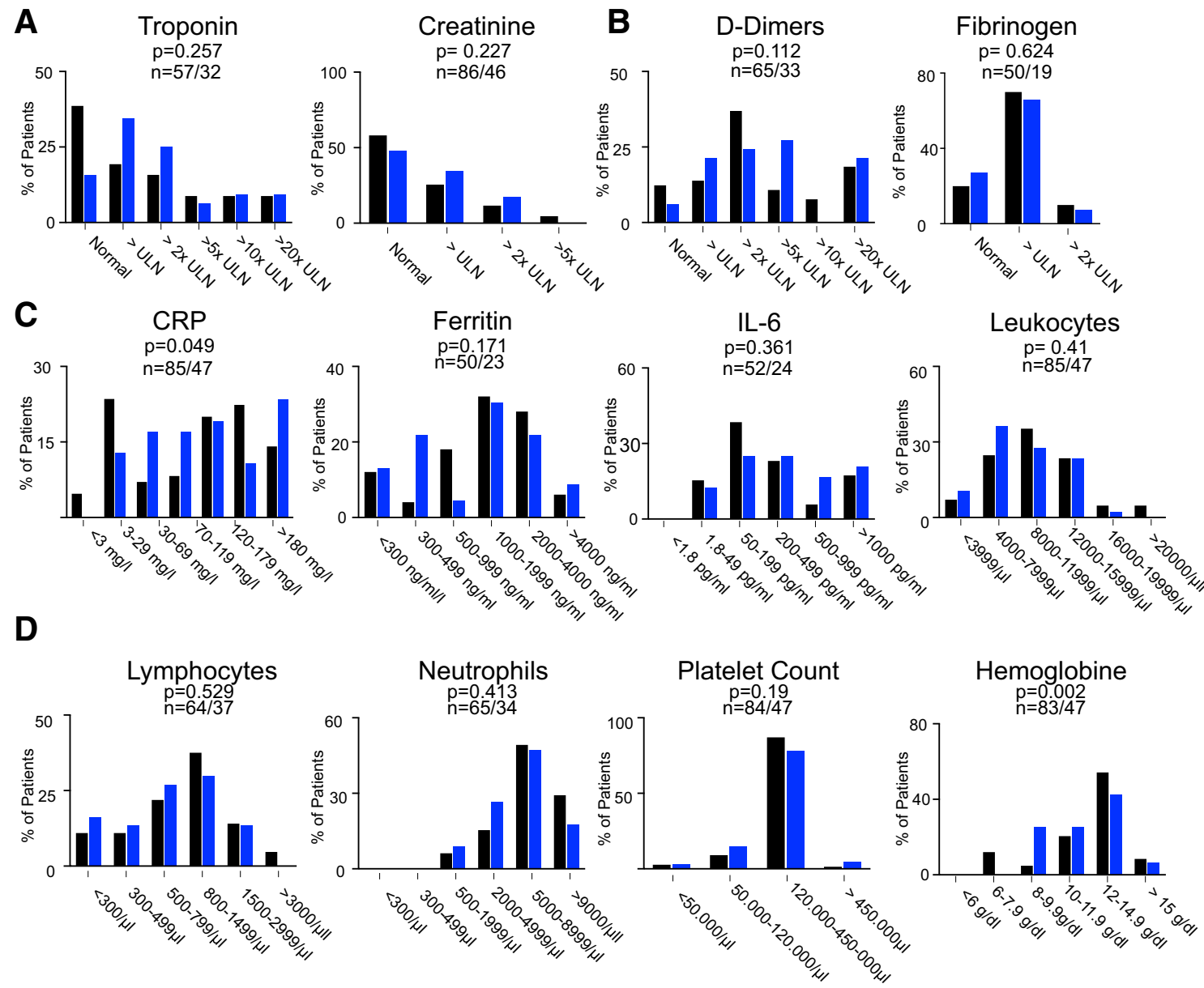

Fig. 3 Serum levels of Troponin and Creatinine (a), D-dimers and Fibrinogen (b), CRP, IL-6, Ferritin, and WBC (c), and Lymphocyte and neutrophil counts, platelet count, and hemoglobin (d) in patients who presented in the critical phase with no cardiovascular comorbid- ity (black bars) and cardiovascular comorbidity (blue bars). n-numbers in patients without cardiovascular comorbidities compared to patients with cardiovascular disease and $p$ values are depicted in each panel $p<0.001$ ], followed by IL6-levels [OR 1.69 (95\% CI 1.26-2.27); $p<0.013$ ] and CRP [OR 1,32; (95\% CI 1.1-1.58); $p<0.003$ ]. In addition, creatinine and higher total leukocyte count and neutrophil levels as well as lower levels of hemoglobin and platelets where independent predictors of mortality in multivariate analysis. Here, all other markers lost their predictive significance (Fig. 6b).

\section{Discussion}

The results of the present analysis from a very large European Registry on COVID-19, the LEOSS registry, for the first time demonstrate that patients with cardiovascular comorbidities exhibit increased thrombo-inflammatory 


\section{Biomarkers in COVID-19 Complicated Phase}

- No CV Comorbidity, $n=430$

CV Comorbidity, $n=211$
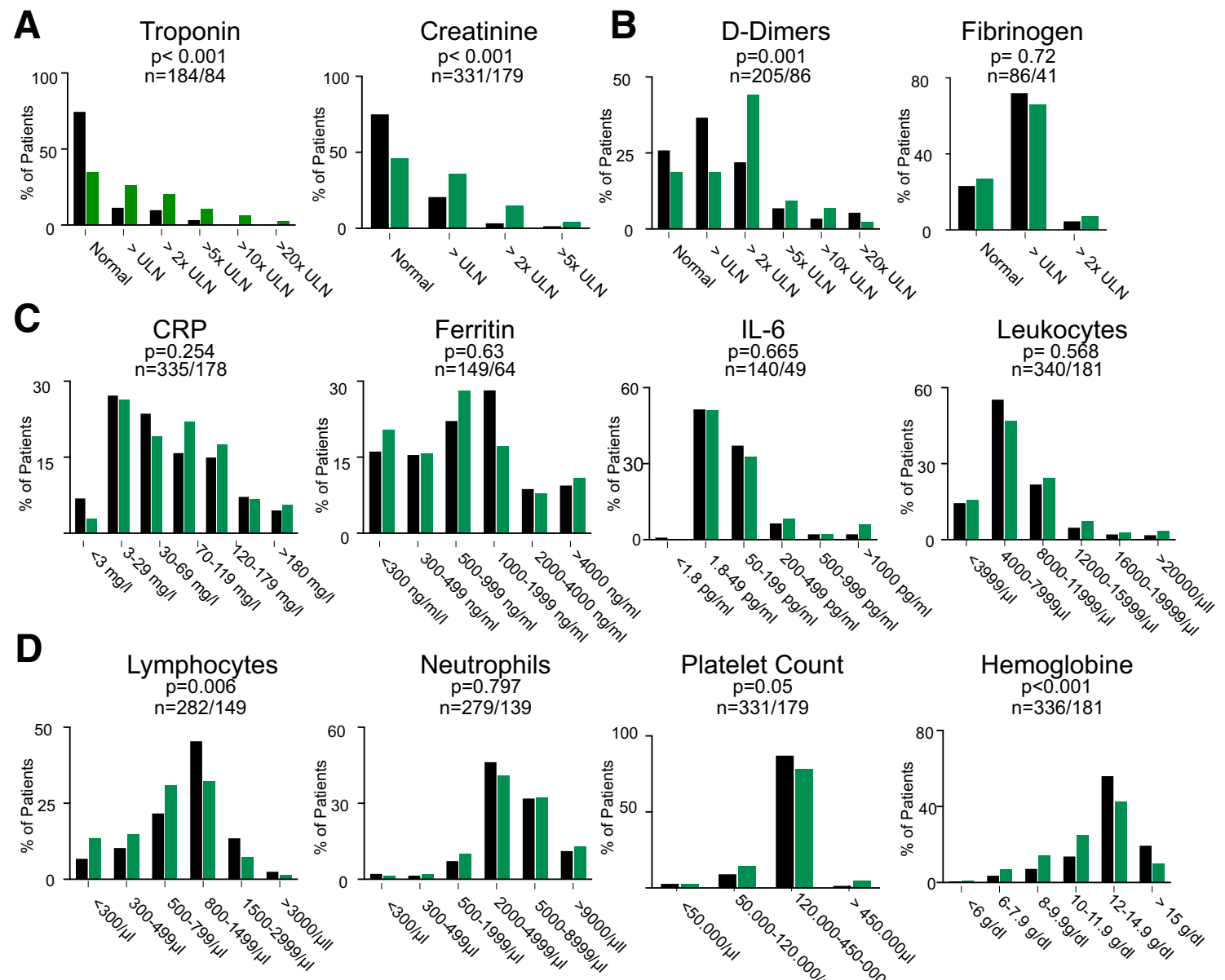
Platelet Count $p=0.05$
$n=331 / 179$
Hemoglobine $p<0.001$
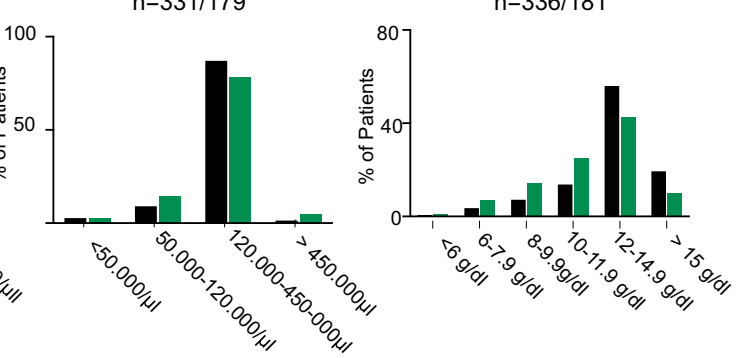

Fig. 4 Serum levels of Troponin and Creatinine (a), D-dimers and Fibrinogen (b), CRP, IL-6, Ferritin and WBC (c), Lymphocyte and neutrophil counts, platelet count, and hemoglobin (d) in patients who presented in the complicated phase with no cardiovascular comorbid- ity (black bars) and cardiovascular comorbidity (green bars). n-numbers in patients without cardiovascular comorbidities compared to patients with cardiovascular disease and $p$ values are depicted in each panel

microvascular thrombi in COVID-19 [10]. In accordance with previous reports, the results of the present study document that thrombo-inflammatory markers in blood were associated with disease progression and mortality [4, 11, 12]. Most importantly, however, patients with cardiovascular comorbidities defined as coronary artery disease, chronic heart failure, and atrial fibrillation displayed an elevated thrombo-inflammatory state as evidenced by significantly elevated levels of D-dimers, CRP, IL-6, and neutrophils already at admission in the uncomplicated phase of COVID19. On multivariate analysis in addition to troponin, IL-6 and CRP serum levels remained significant predictors of mortality in patients initially admitted to the hospital in a severity and multi-organ involvement due to inflammatory 


\section{Biomarkers in COVID-19 Uncomplicated Phase}

- No CV Comorbidity, $\mathrm{n}=1024$ CV Comorbidity, $n=319$
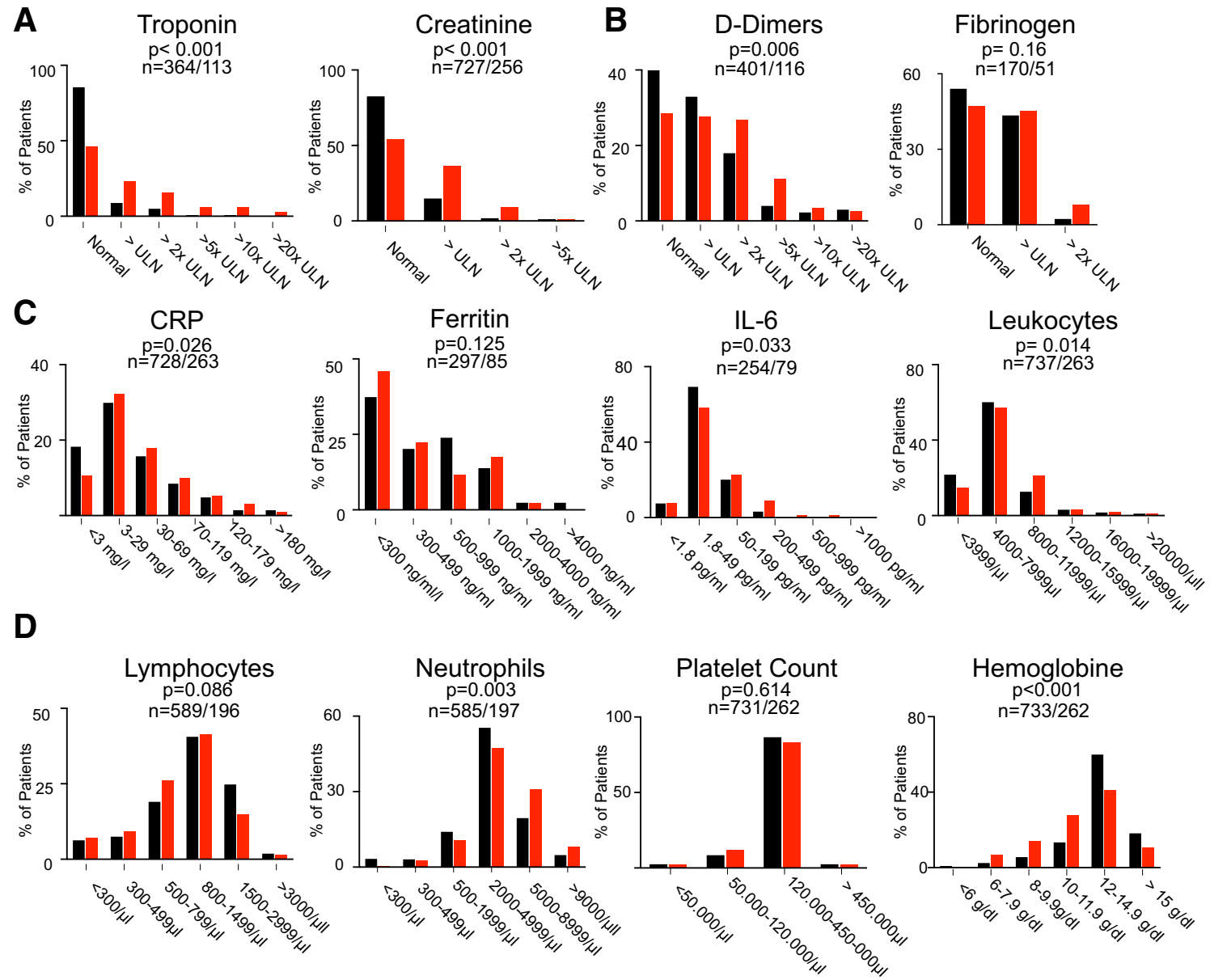

Fig. 5 Serum levels of Troponin and Creatinine (a), D-dimers and Fibrinogen (b), CRP, IL-6, Ferritin, and WBC (c), and Lymphocyte and Neutrophil counts, platelet count, and hemoglobin (d) in patients who presented in the uncomplicated phase with no cardiovascular comorbidity (black bars) and cardiovascular comorbidity (red bars n-numbers in patients without cardiovascular comorbidities compared to patients with cardiovascular disease and $\mathrm{p}$ values are depicted in each panel

but also increasingly described in COVID-19 [13-15]. Activated inflammatory cells are increasingly recognized to contribute to cardiovascular disease [16]. Atherosclerosis, heart failure, and atrial fibrillation are well established to be associated with elevated inflammation markers including IL-6 [17, 18]. Moreover, the recent discovery of somatic mutations in hematopoietic cells ("clonal hematopoiesis of indeterminate potential (CHIP)"), which is associated with increased aggressiveness of inflammatory cells and is significantly enriched in patients with cardiovascular disease, does provide a further link connecting aging and inflammation in cardiovascular disease [19]. Indeed, our recent single-cell 


\section{Forrest plots of uni- and multivariate analysis for mortality of patients who presented in a non-critical phase}
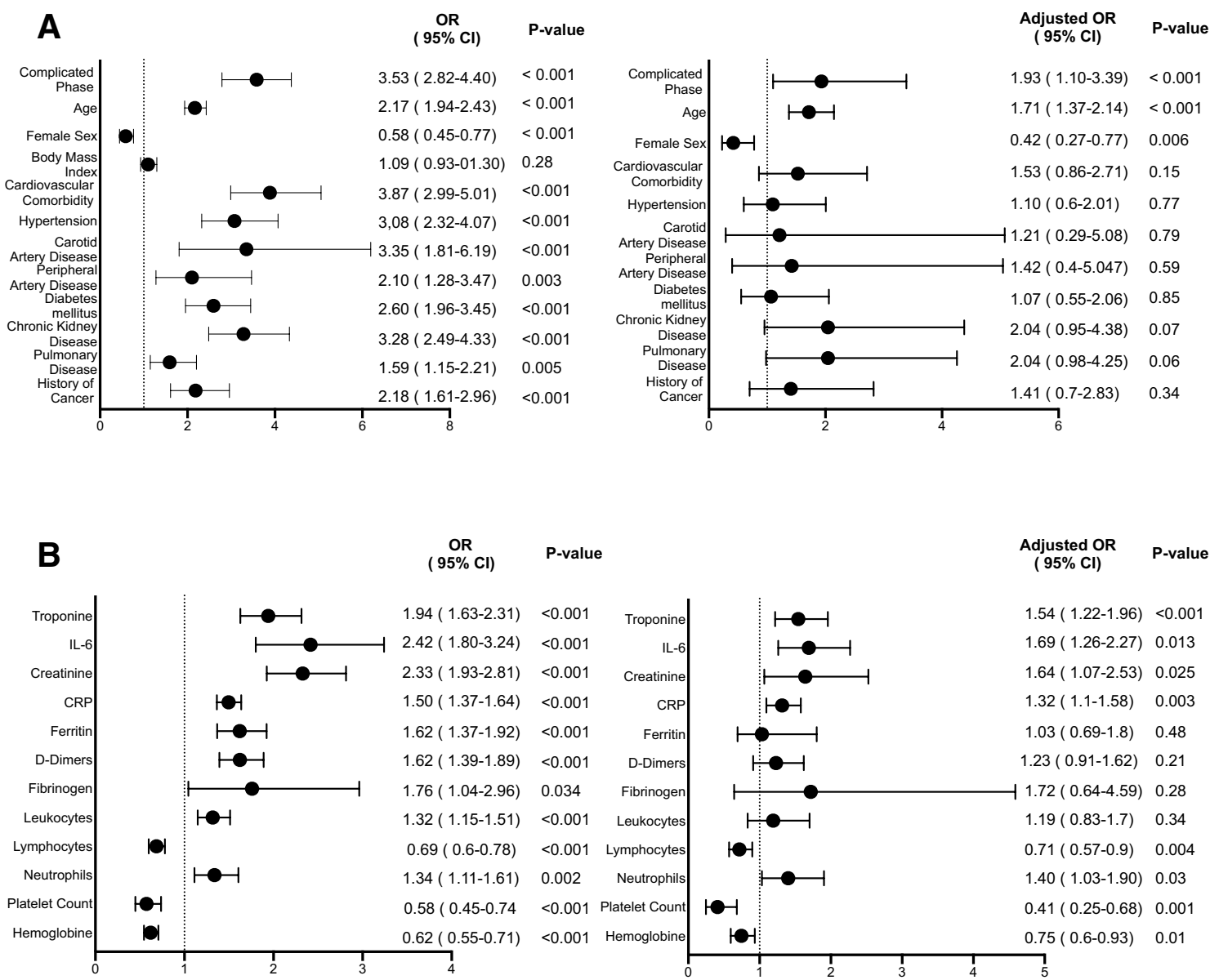

Fig. 6 Forrest plots of univariate and multivariate analysis of clinical indicators (a) and biomarker alterations (b) for mortality in patients who were initially in a non-critical phase considering disease severity (uncomplicated or complicated) at baseline

RNA-sequencing study revealed that monocytes of patients with cardiovascular disease carrying CHIP driver mutations are primed for exuberant inflammatory responses and IL-6 production [20]. Taken together, patients with cardiovascular disease are sensitized for inflammatory activation, which may contribute to worse clinical outcomes following infection with SARS-CoV-2.

Importantly, IL-6 is not only an important biomarker and possible target for cardiovascular morbidity and mortality, but may also serve as a therapeutic target in patients with COVID-19 [21]. Indeed, clinical trials assessing the benefits of inflammatory cytokine blockade targeting IL-6 and its upstream mediator IL-1ß are in progress [9]. Given the heterogeneous preliminary results so far in trials applying IL-6 blockade in more severe cases of COVID-19 [22, 23], the results of the present study may suggest to elucidate the specific role of IL-6 blockade in uncomplicated COVID19 patients with cardiovascular comorbidities. Likewise, in light of the observed enhanced intravascular coagulation even during the uncomplicated phase of COVID-19 in patients with cardiovascular comorbidities, more aggressive anticoagulation treatment strategies may be warranted in these patients, as suggested in a recent retrospective analysis [24].

\section{Limitations}

By virtue of the design of the LEOSS registry to anonymously capture patient data, we cannot provide absolute values for the individual biomarkers, which were divided into different categories. In addition, we do not have sequential 
Table 1 Baseline characteristics of patients in an uncomplicated, complicated or critical phase of COVID-19 disease at day of an initial positive test result for SARS-CoV-2

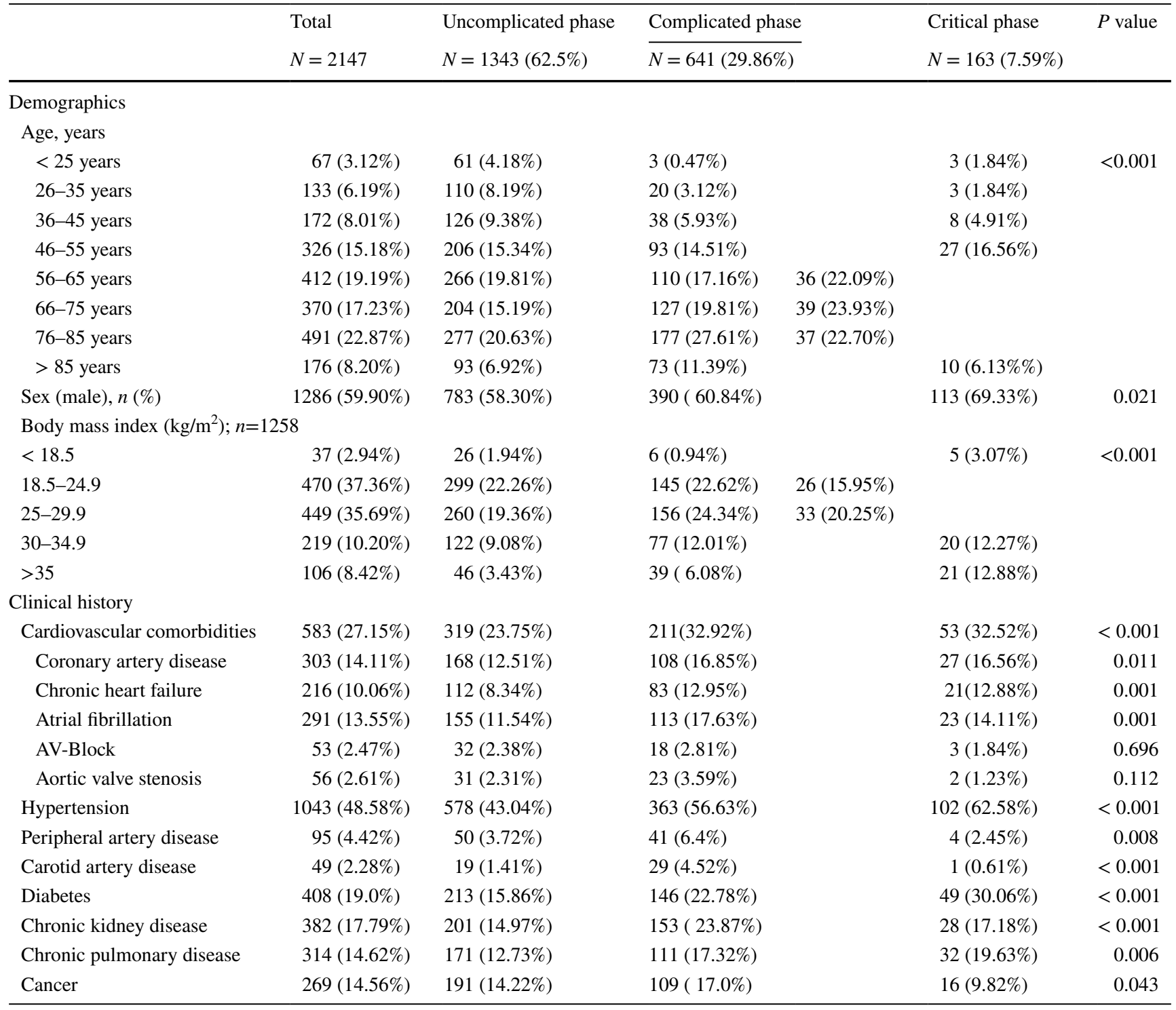

biomarker measurements. Thus, we cannot comment on the possible role of dynamic alterations of individual biomarkers in the process of disease progression. However, we believe that it is important to risk stratify COVID-19 patients at the time of initial presentation to facilitate the induction of specific treatment strategies. Finally, cause of death was not analyzed in the present cohort.

In summary, patients with cardiovascular comorbidities are characterized by an enhanced thrombo-inflammatory activation already during the uncomplicated phase of COVID-19, which associates with worse outcome and increased mortality. Future studies should address a potential benefit of targeted anti-inflammatory therapy and aggressive anticoagulation in patients with cardiovascular comorbidities infected with SARS-CoV-2 already during the uncomplicated phase of COVID-19.

Acknowledgements We thank the following study sites for enrolling patients in LEOSS. Study sites that contributed at least 5 per mille to the analysis of this study were: Stefan Borgmann ( Klinikum Ingolstadt), Lukas Tometten (Hospital Ernst von Bergmann, Potsdam), Kai Wille (Johannes Wesling Hospital, Minden), Beate Gruener ( Universitätsklinikum Ulm), Simon Weidlich (Klinikum Rechts der Isar, München), Siegbert Rieg (Universitätsklinikum Freiburg), Matthias Wettstein (Klinikum Passau), Claudia Reichle (Tropenklinik Tübingen), Maria Ruethrich (Universitätsklinikum Jena), Frank Hanses (Universitätsklinikum Regensburg), Hartwig Klinker (Universitätsklinikum Würzburg), Lukas Eberwein (Klinikum Leverkusen), Christiane Wirtz (Krankenhaus Wuppertal), Katja de With (Universitätsklinikum Dresden), Julia Fürst (Universitätsklinikum Erlangen), Martin Hower (Klinikum Dortmund), Norma Jung (Universitätsklinikum Köln), Sebastian Dolff (Universitätsklinikum Essen), Martin Bergwelt 
(LMU Klinikum München), Timo Brandenburger (Universitätsklinikum Düsseldorf), Maria Vehreschild (Universitätsklinikum Frankfurt), Uta Merle (Universitästklinikum Heidelberg), Dominic Rauschning (Bundeswehrkrankenhaus Koblenz), Rüdiger Lange (Deutsches Herzzentrum München), Christian Hohmann (Klinikum Bremen-Mitte), Beate Schultheiss (Marienhospital Herne), Sabine Jordan (Universitätsklinikum Hamburg Eppendorf), Kerstin Werth (Malteser Krankenhaus St. Franziskus Hospital Flensburg), Susanne Rueger (Klinik München), Katja Rothfuss (Robert-Bosch-Krankenhaus, Stuttgart), Jacob Nattermann (Universitätsklinikum Bonn), Marc Bota (Bethesda Klinikum Bergedorf), Anja Walter-Ruf (Krankenhaus St-Joseph-Stift Dresden), Kerstin Hellwig (Katholisches Klinikum Bochum), Berit Amelie Bretthauer ( Universitäts Herzzentrum Freiburg), Annika Ritter (Klinikum Osnabrück), Jürgen Prattes (Medizinische Universität Graz), Leoss Investigators (Klinikum Karlsruhe), Janina Traut (Universitätsklinikum Gießen und Marburg), Fabio Lizzi (Universitätsklinikum des Saarlandes), and Daniel Droemann ( Universitätsklinikum SchleswigHolstein, Standort Lübeck).

Funding Open Access funding enabled and organized by Projekt DEAL. The study was supported by the German Center for Infection Research, the Pitzer Foundation to J.V., and the German Center for Cardiovascular Research DZHK, Berlin, Germany to A.B.

\section{Compliance with ethical standards}

Conflict of interest The authors declare that they have no conflict of interest.

Open Access This article is licensed under a Creative Commons Attribution 4.0 International License, which permits use, sharing, adaptation, distribution and reproduction in any medium or format, as long as you give appropriate credit to the original author(s) and the source, provide a link to the Creative Commons licence, and indicate if changes were made. The images or other third party material in this article are included in the article's Creative Commons licence, unless indicated otherwise in a credit line to the material. If material is not included in the article's Creative Commons licence and your intended use is not permitted by statutory regulation or exceeds the permitted use, you will need to obtain permission directly from the copyright holder. To view a copy of this licence, visit http://creativecommons.org/licenses/by/4.0/.

\section{References}

1. Böhm M, Frey N, Giannitsis E, Sliwa K, Zeiher AM (2019) Coronavirus Disease 2019 (COVID-19) and its implications for cardiovascular care: expert document from the German Cardiac Society and the World Heart Federation. Clin Res Cardiol. https ://doi.org/10.1007/s00392-020-01656-3

2. Wu Z, McGoogan JM (2020) Characteristics of and important lessons from the Coronavirus Disease 2019 (COVID-19) outbreak in China: summary of a report of 72314 cases from the Chinese center for disease control and prevention. JAMA 323:1239-1242

3. Richardson S, Hirsch JS, Narasimhan M, Crawford JM, McGinn T, Davidson KW, TNCOVID-1RC, Barnaby DP, Becker LB, Chelico JD, Cohen SL, Cookingham J, Coppa K, Diefenbach MA, Dominello AJ, Duer-Hefele J, Falzon L, Gitlin J, Hajizadeh N, Harvin TG, Hirschwerk DA, Kim EJ, Kozel ZM, Marrast LM, Mogavero JN, Osorio GA, Qiu M, Zanos TP (2020) Presenting characteristics, comorbidities, and outcomes among 5700 patients hospitalized with COVID-19 in the New York City area. JAMA 323:2052-2059
4. Zhou F, Yu T, Du R, Fan G, Liu Y, Liu Z, Xiang J, Wang Y, Song B, Gu X, Guan L, Wei Y, Li H, Wu X, Xu J, Tu S, Zhang Y, Chen H, Cao B (2020) Clinical course and risk factors for mortality of adult inpatients with COVID-19 in Wuhan, China: a retrospective cohort study. Lancet 395:1054-1062

5. Shi S, Qin M, Cai Y, Liu T, Shen B, Yang F, Cao S, Liu X, Xiang Y, Zhao Q, Huang H, Yang B, Huang C (2020) Characteristics and clinical significance of myocardial injury in patients with severe coronavirus disease 2019. Eur Heart J 41:2070-2079

6. Inciardi RM, Adamo M, Lupi L, Cani DS, Di Pasquale M, Tomasoni D, Italia L, Zaccone G, Tedino C, Fabbricatore D, Curnis A, Faggiano P, Gorga E, Lombardi CM, Milesi G, Vizzardi E, Volpini M, Nodari S, Specchia C, Maroldi R, Bezzi M, Metra M (2020) Characteristics and outcomes of patients hospitalized for COVID-19 and cardiac disease in Northern Italy. Eur Heart J 41:1821-1829

7. Lala A, Johnson KW, Januzzi JL, Russak AJ, Paranjpe I, Richter F, Zhao S, Somani S, Van Vleck T, Vaid A, Chaudhry F, De Freitas JK, Fayad ZA, Pinney SP, Levin M, Charney A, Bagiella E, Narula J, Glicksberg BS, Nadkarni G, Mancini DM, Fuster V, Mount SCIC (2020) Prevalence and impact of myocardial injury in patients hospitalized with COVID-19 infection. J Am Coll Cardiol 76:533-546

8. Jakob C, Borgmann S, Duygu F, Behrends U, Hower M, Merle U, Friedrichs A, Tometten L, Hanses F, Jung N, Rieg S, Wille K, Grüner B, Klinker H, Gersbacher-Runge N, Hellwig K, Eberwein L, Dolff S, Rauschning D, von Bergwelt-Baildon M, Lanznaster J, Strauss R, Trauth J, de With K, Ruethrich M, KLueck C, Nattermann J, Tscharntke L, Pilgram L, Fuhrmann S, Classen A, Stecher M, Schons M, Spiner C, Verhreschild JJ, (2020) First results of the "Lean European Open Survey on SARS-CoV-2-Infected Patients (LEOSS)." Infection. https://doi.org/10.1007/s15010-020

9. Merad M, Martin JC (2020) Pathological inflammation in patients with COVID-19: a key role for monocytes and macrophages. Nat Rev Immunol 20:355-362

10. LA Nicolai L, Brambs S, Kaiser R, Weinberger T, Weigand M, Muenchhoff M, Hellmuth JC, Ledderose S, Schulz H, Scherer C, Rudeliius M, Zoller M, Höchter D, Keppler O, Teupser D, Zwißler B, Bergwelt-Baildon M, Kääb S, Massberg S, Pekayvaz K, Stark K (2020) Immunothrombotic dysregulation in COVID-19 pneumonia is associated with respiratory failure and coagulopathy. Circulation 142:1176-1189

11. Ruan Q, Yang K, Wang W, Jiang L, Song J (2020) Clinical predictors of mortality due to COVID-19 based on an analysis of data of 150 patients from Wuhan, China. Intensive Care Med 46:846-848

12. Chen G, Wu D, Guo W, Cao Y, Huang D, Wang H, Wang T, Zhang X, Chen H, Yu H, Zhang X, Zhang M, Wu S, Song J, Chen T, Han M, Li S, Luo X, Zhao J, Ning Q (2020) Clinical and immunological features of severe and moderate coronavirus disease 2019. J Clin Invest 130:2620-2629

13. Zhang Y, Xiao M, Zhang S, Xia P, Cao W, Jiang W, Chen H, Ding $\mathrm{X}$, Zhao H, Zhang H, Wang C, Zhao J, Sun X, Tian R, Wu W, Wu D, Ma J, Chen Y, Zhang D, Xie J, Yan X, Zhou X, Liu Z, Wang J, Du B, Qin Y, Gao P, Qin X, Xu Y, Zhang W, Li T, Zhang F, Zhao Y, Li Y, Zhang S (2020) Coagulopathy and antiphospholipid antibodies in patients with Covid-19. N Engl J Med 382:e38

14. Varga Z, Flammer AJ, Steiger P, Haberecker M, Andermatt R, Zinkernagel AS, Mehra MR, Schuepbach RA, Ruschitzka F, Moch $\mathrm{H}$ (2020) Endothelial cell infection and endotheliitis in COVID19. Lancet 395:1417-1418

15. Ackermann M, Verleden SE, Kuehnel M, Haverich A, Welte T, Laenger F, Vanstapel A, Werlein C, Stark H, Tzankov A, Li WW, Li VW, Mentzer SJ, Jonigk D (2020) Pulmonary vascular endothelialitis, thrombosis, and angiogenesis in Covid-19. N Engl J Med 183:120-128 
16. Nahrendorf M (2018) Myeloid cell contributions to cardiovascular health and disease. Nat Med 24:711-720

17. Hijazi Z, Oldgren J, Siegbahn A, Granger CB, Wallentin L (2013) Biomarkers in atrial fibrillation: a clinical review. Eur Heart J 34:1475-1480

18. Ridker PM, Lüscher TF (2014) Anti-inflammatory therapies for cardiovascular disease. Eur Heart J 35:1782-1791

19. Libby P, Sidlow R, Lin AE, Gupta D, Jones LW, Moslehi J, Zeiher A, Jaiswal S, Schulz C, Blankstein R, Bolton KL, Steensma D, Levine RL, Ebert BL (2019) Clonal hematopoiesis: crossroads of aging, cardiovascular disease, and cancer: JACC review topic of the week. J Am Coll Cardiol 74:567-577

20. Abplanalp WT, Mas-Peiro S, Cremer S, John D, Dimmeler S, Zeiher AM (2020) Association of clonal hematopoiesis of indeterminate potential with inflammatory gene expression in patients with severe degenerative aortic valve stenosis or chronic postischemic heart failure. JAMA Cardiol. https://doi.org/10.1001/jamacardio .2020 .2468

21. Guzik TJ, Mohiddin SA, Dimarco A, Patel V, Savvatis K, MarelliBerg FM, Madhur MS, Tomaszewski M, Maffia P, D’Acquisto F, Nicklin SA, Marian AJ, Nosalski R, Murray EC, Guzik B, Berry C, Touyz RM, Kreutz R, Wang DW, Bhella D, Sagliocco O, Crea
F, Thomson EC, McInnes IB (2020) COVID-19 and the cardiovascular system: implications for risk assessment, diagnosis, and treatment options. Cardiovasc Res 116:1666-1687

22. Patel K, Gooley TA, Bailey N, Bailey M, Hegerova L, Batchelder A, Holdread H, Dunleavy V, Downey T, Frisvold J, Megrath S, Pagarigan K, Szeto J, Rueda J, Islam A, Maree C, Nyatsatsang S, Bork SE, Lipke A, O’Mahony DS, Wagner T, Pulido J, Mignone J, Youssef S, Hartman M, Goldman JD, Pagel JM (2020) Use of the IL-6R antagonist tocilizumab in hospitalized COVID-19 patients. J Intern Med. https://doi.org/10.1111/joim.13163

23. Strohbehn GW, Heiss BL, Rouhani SJ, Trujillo JA, Yu J, Kacew AJ, Higgs EF, Bloodworth JC, Cabanov A, Wright RC, Koziol A, Weiss A, Danahey K, Karrison TG, Edens CC, Ventura IB, Pettit NN, Patel B, Pisano J, Strek ME, Gajewski TF, Ratain MJ, Reid PD. COVIDOSE: Low-dose tocilizumab in the treatment of Covid-19. medRxiv. 2020. https://doi. org/10.1101/2020.07.20.20157503

24. Paranjpe I, Fuster V, Lala A, Russak A, Glicksberg BS, Levin MA, Charney AW, Narula J, Fayad ZA, Bagiella E, Zhao S, Nadkarni GN (2020) Association of treatment dose anticoagulation with in-hospital survival among hospitalized patients with COVID-19. J Am Coll Cardiol 76:122-124 